Paulina Irene Salinas Meruane

Escuela de Periodismo de la Universidad Católica del Norte, Chile

\title{
Los discursos masculinos como dispositivos de control y tensión en la configuración del liderazgo y empoderamiento femenino
}

\begin{abstract}
Resumen: En el presente artículo se exponen algunos de los antecedentes centrales del proyec to de investigación "Los Discursos de la Masculinidad": El Empoderamiento Femenino desde el Análisis C ritic o del Disc urso. ${ }^{1}$ Utilizando un enfoque cualitativo, se desarrollaron entrevistas a biertas con el objetivo de comprender el signific a do que le a signan los hombres al liderazg o femenino. Los discursos fueron analizados en base a la Teoría Fundamentada de Anselm Strauss y J uliet Corbin (2003). ${ }^{2}$ Los resulta dos muestran que los varones c oincid en en términos generales respec to a los cambios que ha habido en los roles de género, en que las mujeres tienen y deben tener más oportunidades, en que hay una mayor incorporación de éstas en el mercado laboral y en la necesidad de tener relaciones más equitativas al interior de la familia. Así mismo, emergen tensiones que configuran el malestar de la masculinidad, traspasando la esfera íntima y c uestionando las rela ciones de intergénero ac tuales. Ta mbién hay disc ursos más progresistas en los varones, que se aventuran hacia un reconocimiento de las capacidades y roles que han id o asumiendo las mujeres en el espacio público, a unque se trata de un reconocimiento vigilante, ambivalente, mediatizado por una percepción de confianza/desconfianza, eficiencia/ incompetencia, hacia lo que ellas son capaces de lograr.
\end{abstract}

Palabras claves: empoderamiento; transformaciones; genero

Copyright 2007 by Revista Estudos Feministas.

${ }^{1}$ Investigación realizada entre el 2005 y 2006 y que contó con el financiamiento de la Dirección General de Investigación y Postgrado de la Universidad Ca tólic a del Norte, Antofagasta. 2 STRAUSS y CORBIN, 2003.

2 STRAUSS Y CORBIN, 2003.

\section{Introducción}

En el presente artíc ulo se exponen a lgunos ha llazgos obtenidos en el proyecto de investigación: Los Discursos de la Masculinidad: El Empoderamiento Femenino desde el Aná lisis C rític o del Disc urso, rea liza do el 2006. Este tra ba jo se enmarca en una línea investigativa que se desarrolla desde el 2003, periodo en el que se abordó la comprensión del liderazgo de las mujeres en organizaciones de la I y II región de Chile. Dicho trabajo permitió describirel liderazgo femenino e identific arforta lezasy debilidades en el ejerc ic io 
${ }^{3}$ Norman FAIRCLOUGH y Ruth WODAK, 2000. del mismo. Luego nos platea mosa va nzaren la comprensión de este fenómeno, teniendo en cuenta dos premisas centrales: la primera, que las preguntas acerca de las mujeres implica comprender la perspectiva de los varones, ya que el género es un sistema de interacciones socia les y no un sistema de compartimientos estancos, es decir, lo que afecta a unasimplica a otros. Y la segunda, referida a concebirel lenguaje y el habla como una foma de práctica social, es decirse trata de desentrañar la relación dia léctic a entre un suceso discursivo particular y las situaciones, instituciones y las estructuras socia les que lo enmarcan. ${ }^{3}$

En consecuencia, a naliza r la práctica discursiva de los hombres sobre el liderazgo y empoderamiento femenino es un factor significativo en la comprensión subjetiva de este fenómeno y ayuda a develar el lenguaje como productor y reproductor de las relaciones de poder desiguales.

Dicho esto, el artículo se centra en analizar los signific a dos que le a signa n los hombres al empodera miento femenino, las tensiones, a mbigüedades y mecanismos de control que tienen respecto a las transformaciones en el ejercicio del poder femenino. Nos interesó profundizar desde la perspectiva masculina, ¿cómo perciben los va rones el poderpersonal que ha n a lc anza do las mujeres?, ¿hay transgresiones y/o reivindicaciones en el empoderamiento femenino?, ¿qué obstáculos reconocen ellos en este proceso? La perspectiva de a ná lisis desde los disc ursos masculinos se centra en la búsqueda de una comprensión holística del fenómeno del liderazgo y participación femenina, entendiendo la perspectiva de género como una categońa relacionalde todos los procesosy fenómenos sociales, y no sólo como un tema que se constriñe a la configuración de las identida des y roles.

Este estudio presenta algunos adelantos en este sentido, ya que se plantea la comprensión de los aspectos subjetivos relacionados a la conformación del liderazgo, teniendo presente que se han producido transformaciones significativas, vinculadas con el cuestionamiento que elaboran los varones acerca de las demandas que se les hace como hombres, lo que representa una expresión de malestar o una cierta perplejidad que se extienden más allá de la esfera íntima y que los interpela en el ejercicio de roles en tanto hombres, padres, parejas, amigos.

\section{El contexto discursivo}

La producción y comprensión del discurso implica tomar en cuenta el contexto, y particularmente en lo que respecta al empoderamiento de las mujeres en América 
${ }^{4}$ Ximena MACHICAO Barbery, 2006.

${ }^{5}$ PNUD, 2004; INE, 2002; OIT, 2000.

\footnotetext{
${ }^{6}$ J osé OLAVARRÍA, 2001b, p. 31.
}

latina debe hacerse referencia al elemento sincrónico del proceso, ya que efectivamente se han producido transformaciones importantes desde la segunda mitad del siglo XX en adelante. Tanto en América Latina como el Caribe se observa una a pertura política que se traduce en una mayor incorporación de las mujeresa los espacios de poder, en cargos de ministras en Chile, Argentina, Brasil, Bolivia y Uruguay; en el área legislativa, judicial, en los gobiemos munic ipales o proyec tándose como candidatas a la presidencia o vicepresidencia, como fue el caso de México y Costa Rica, lo que junto a la larga historia que tienen luchando por una mayor participación y representación política, dan cuenta de que se han producido transformaciones en favor de una ruptura de la hegemonía masculina en el ejercicio del poder polític $0 .{ }^{4}$

En esta dirección, en marzo 2006 se marca un hito en la historia política del país: por primera vez una mujer logra ser elegida presidenta de la república, siendo este hecho en si mismo un avance sin precedentes para las mujeres, y que sólo el a nálisis posterior de la historia podrá dimensionar en toda su magnitud. Sin embargo, instalado el gobiemo de Michelle Bachelet, es oportuno analizar la conflictiva relación de las mujeres y el poder y las reivindicaciones y transgresiones que esta analogía provoca.

En este sentido, si presentan algunos antecedentes preliminares para comprender la dimensión de este fenómeno, y si apuntan a una transformación de loscódigos culturales del país y a una democratización real de la soc iedad, o esta mos presencia ndo a lgunos cambios para que todo permanezca igual. Diversos estudios coinciden favorablemente en que en Chile se ha producido un aumento gradual tanto de la participación como del liderazgo femenino en las distintas áreas del quehacer nacional. Porejemplo en elámbito laboral, la incorporación de las mujeres experimentó, durante la década de los mil novecientos noventa, un incremento sustantivo desde un $28,06 \%$ en 1992 a un $35,5 \%$ en el año 2002 , lo cual marca un incremento superior a 7 puntos porcentuales. ${ }^{5}$

También en la política un enclave eminentemente masculino ha sido levemente modificado, con la inclusión de la paridad como principio articulador del poder ejecutivo - léase ministerios, intendencias, gobemaciones.

En cambio, se constatan resistencias en sectores como las organizaciones sindicales, las que siguen controladas por los varones que son quienes articulan los espacios y redes de homo sociabilidad y, en general, lo mismo se observa en los puestos de mayor jerarquía pública. ${ }^{6}$ 
7 Teresa VALDÉS y Mariza WEINSTEIN, 1993; OIga RUIZ, Sandra SOLANO y Claudia Zapata, 1998; SERNAM, 2000; GIM, 2002; VALÉS, 2001; Paulina SALINAS, 2002; PNUD, 2004.

${ }^{8}$ PNUD, 2004, p. 87.

${ }_{9}^{9}$ PNUD, 2004, p. 17.

\footnotetext{
${ }^{10}$ Mala HTUN, 2003.
}

Otros estudios profundizan las complejidades que han experimentado las mujeres en su lucha por alcanzar un mayor protagonismo y reconocimiento en la sociedad. ${ }^{7}$ Esta s investig a c iones devela $n$ la s tensiones que ha $n$ existido entre las mujeres y su acceso a los espacios de poder. Algunos de los argumentos que se arguyen para explicar este fenómeno se relacionan con pesa dos lastres y grandes brechas que caracterizan la distribución de recursos y derechos existentes en la sociedad chilena.

El quinto Informe sobre Desa rrollo Humano identifica dos elementos que frenan un mayor y mejor aprovechamiento de las oportunidades que el país ofrece, y que se relacionan con la insuficiente disposición de podersocial y una desigual distribución del poder de acción personal, ambas afectando la capacidad de los ciudadanos para a provechar las oportunidades y levantando barreras a la a soc ia tividad. ${ }^{8}$

Otras amenazas más imperceptibles se vinculan a la permanencia del statu quo de parte de quienes no quieren ceder poder: el temor a entregar poder a otras personas, especialmente a las mujeres, y a las organizaciones. Hay desc onfianza en las capacidades de esto a c tores para tomar dec isiones. ${ }^{9} \mathrm{O}$ tras resistenc ias son hacia lo que implica promoverla participa ción ciudadana, sea n esta s en inicia tiva scolec tiva so ind ividuales; ha y temor al "desborde", a los pliegos de peticiones, al a umento de expectativas, entre otras.

Por lo tanto, a nalizar los a vances y retrocesos en el proceso de empoderamiento y construcción ciudadana de lasmujeres como sujetos polític os con relevancia social, facilita la comprensión de los cambios en su verdadera dimensión como una manera de visualizar los desafíos que tiene la sociedad chilena en su conjunto, entendiend o que los avances en la consolidación de la democracia están entramados al desarrollo sostenible y a un crecimiento económico equitativo.

Sin embargo, la mayor libertad política y la promoción de mercados más liberalizados no han desencadenado directamente avances sustantivos en cuanto a los derechosciudadanos, ni respecto al bienestar económico de la población en su conjunto. De hecho plantea Mala Htun que en la medida que las brechas salariales se incrementan, muchas mujeres en La tinoamérica carecen de las capacidades básicas y permanecerán excluidas de los sectores elegibles para ocuparcargos de mando. ${ }^{10}$

Por ejemplo, no es menor que, no sólo en el país, sino que en el resto del mundo las mujeres, a unque representan más de la mitad del electorado, apenas 
${ }^{11}$ MACHICAO, 2006, p. 20.

12 PNUD, 2004, p. 21.

${ }^{13}$ OLAVARRÍA, 2001b, p. 24.

${ }^{14}$ Robert W. CONNEL, 1998, p. 77. alcanzan al $12,7 \%$ del total de bancadas parlamentarias en el mundo, y que en Améric a Latina no logran el 10\%. Si bien en algunos países como Argentina y Costa Rica muestran log ros importantes, en otras nac iones los prog resos han sido relativos o nulos, a pesar de las medidas de disc rimina ción positiva, que de hec ho no tienen un carácter vinculante a la Constitución Polític as de los Estados. Por lo tanto, su a plicación se encuentra supedita da a la voluntad de los gobiernos de turno. ${ }^{11}$ En este contexto, cabe preguntarse para el caso chileno, en qué medida la pariedad introducida por el gobierno de Bachelet por ejemplo, ¿es una iniciativa que perdurará en el tiempo?, ¿será que la presencia de las mujeresen el poderejecutivo marcará la agenda pública?, ¿cuáles son los obstáculos que enfrentan en los puestos de poder?, ¿la mayor presencia de las mujeres en cargos de representación conlleva modificaciones en los mandatos de género?, es decir son múltiples los cuestionamientos plausibles de hacerse y las sospechas que se generan sobre el tipo de política que se está construyendo en el país. Además, las a lia nza s polític as femeninas no siempre han log ra do resistir con éxito las exigencias partidarias con la inclusión de temas que favorezcan los derechos de las mujeres.

Estas interrogantes permiten abrir la disc usión hacia esferas que hoy día son gravitantes para el desarrollo y la consolidación del sistema democrático. Chile ha tenido logros importantes en materia de indicadores sociales, ha alcanzado un destacado posicionamiento en cuanto al Desarrollo Humano en el contexto latinoamerica no, ${ }^{12}$ a lo que se suma un mejoramiento en la búsqueda de un mayor equilibrio en las relaciones entre hombres y mujeres, producto de la evolución económica, social y cultural de la sociedad chilena y del mundo en general. ${ }^{13}$ Tanto la globalización como los cambios de la economía han potenciado demandas de modemización en el espacio íntimo, cuestionándose las bases de la masculinidad a utoritaria y la fa milia nuclear patria rcal, por perpetua resta última la inequidad, limitar la autonomía, la diversidad y las relaciones democráticas al interior de la familia. Asimismo, los debates respec to a las relaciones de género se están produciendo globalmente. Las relaciones de género forman parte de la estructura de la sociedad mundial y es necesario reconsiderar muchos temas a la luz de este hecho. ${ }^{14}$

Junto a lo anterior, la sociedad chilena también presenta irrefutables fracturas, una inequita tiva distribución de los ingresos, insuficiente política de igualdad de oportunidades, entre otras. En consecuencias, hoy día es una condición sine qua non del desarrollo huma no 
fortalecer la participación ciudadana, entregando más poder a cada uno y a la sociedad en su conjunto.

\section{Estrategia metodológica y corpus}

Como mencionamos anteriormente, la propuesta investiga tiva se inscribe dentro del Aná lisis C ritic o de Disc urso (en adelante $A C D$ ), concebido éste como un enfoque que se ocupa de develar las relaciones de dominación, discriminación, poder y control, tal como se manifiesta a

${ }^{15}$ WODAK, 2003, p. 19

${ }^{16}$ Ron SCOLON, 2003.

${ }^{17}$ Teun A. VAN DIJ K, 1997, p. 15.

${ }^{18}$ Ang elika HENNECKE, 2004, p. 107. ${ }^{19}$ FAIRC LOUGH Y WODAK, 2000.

${ }^{20}$ Lupic inio IÑIG UEZ Rueda, 2003, p. 94. tra vés del lenguaje. En otras palabras plantea Wodak, ${ }^{15}$ el $A C D$ se propone investigar de forma crítica la desigualdad social tal como viene señalada, expresada, constituida, legitimada porlos usos del lenguaje.

El lenguaje acompaña frecuentemente a nuestras acciones, y lasacciones a compañan gran parte de lo que decimos. ${ }^{16}$ Así entonces, el ACD propone analizar críticamente el discurso como una forma de abordar los problemas sociales que se van concadenando en los discursos. Vale decir nos interesó analizar qué y cómo hablan los hombres respec to a las transformaciones en el empoderamiento de las mujeres, cómo discursean sobre loscambiosque se han experimentado ellas en la sociedad, qué tensiones se generan, y cómo viven esta experiencia tanto en el ámbito público como privado.

El ACD es la denomina ción genérica que se aplica a un planteamiento especial dedic ado a estudiar los textos y el habla y que emergen de la crítica lingüística, semiótica, $y$, en general, del modo sociopolítico consciente y oposicionista en que se estudia el lenguaje, el disc urso y la comunicación. ${ }^{17}$ La ampliación de la lingüística del texto pragmático - comunic a tiva por el Análisis Crítico del Discurso consiste en que ésta le pemite visua liza r la relevancia social y política de los textos, faculta mostrar una dimensión de significado que sobrepasa el encasillamiento a un marco situacional, concreto y actual. ${ }^{18}$ Es una forma de intervenir en la práctica social y en las relaciones sociales. ${ }^{19}$ Es considera do una práctica tridimensional, en el sentid o que la práctica analítica opera en tres dimensiones: a) el discurso en tanto texto, b) el discurso como práctica discursiva enmarcado en una situa ción social concreta, y el c) el discurso como un ejemplo de práctica social, que no sólo expresa o refleja identidades, prácticas, relaciones, sino que la constituye y conforma. ${ }^{20}$

A la luz de esta propuesta analític a se desarrolló un estudio descriptivo con fines interpretativos, es decir, una propuesta investigativa eminentemente cualitativa, en la que se concibe la realidad social como subjetiva, múltiple y cambiante, como resultado de una construcción de los 
${ }^{21}$ J osé Ignacio RUIZ, 1999; Miguel S. VAШES, 2000; María Paz SADÍN Esteban, 2003.

22 STRAUSS y CORBIN, 2002.

${ }^{23}$ RUIZ, 1999, p. 64.

${ }^{24}$ STRAUSS y CORBIN, 2002. sujetos partic ip antes que se encuentran en interac c ión con otros miembros de la sociedad. ${ }^{21}$

El corpus del estudio estuvo conformado por los discursos de 59 hombres. En la primera fase investigativa se aplicaron 20 entrevistas individuales en profundidad, durante el 2005. Esta etapa se desarrolló en forma secuencial (comparación constante), ${ }^{22}$ de tal manera que una vezque la primera entrevista fue realizada, se procedió a su trascripción y a nálisis preliminar; de este modo la segunda entrevista incorporó los aportes de la primera y así suc esivamente. Luego en una segunda fase, durante el 2006, se entrevistaron 39 hombres en cuatro entrevistas grupales, de tal manera de triangular la información obtenida en la modalidad individual y así acceder al discurso social de los varones.

Para seleccionar a los entrevistados se utilizó un muestreo cua lita tivo intencional, ${ }^{23}$ busc ando distintos representantes del universo masculino en la región. Se entrevistó en la modalidad individual a varones, con distintos niveles socioeducacionales, nueve profesionales universitarios, cinco con forma ción téc nic a, tres estudia ntes universita rios y trescon cuarto a ño medio rendido. Lasedades fluctuaron entre los 19 y los 63, con edad promedio de 40 años.

En la primera entrevista grupal participaron 10 varones, cuyas edades fluctúan entre 21 y 46, con un promedio de 30 años, todos tienen cuarto año medio rendido y se desempeñan como operarios en la empresa C.S.M, Tecnología Ltda. de Antofagasta. En la segunda, participaron 14 varones, cuyas edades fluctúan entre 39 y 56 años, y 46 años de edad promedio, el nivel de escolaridad de los participantes es de enseñanza básica (5), enseña nza media (6) y a lgunos tiene forma ción téc nica (3). En la tercera, participaron 7 varones, cuyas edades fluctúan entre los 32 y 49 años, y 38 años de edad promedio. Se desempeñan como a uxiliares de servicio en el liceo Experimental Artístic o de Anto fa ga sta. El nivel de esc ola ridad de los entrevistados es enseñanza básica completa (3), enseñanza media incompleta (3) y completa (1). Y en la cuarta entrevista participaron 8 varones que se desempeñan como personal en la Cárcel de Hombres de la ciudad de Antofagasta (gendarmes y vigilantes), cuyas edades fluctúan entre 19 y 43 años, con 29 años de edad promedio. Todos poseen cuarto año medio rendido.

El a ná lisis de los da tos complementó dos propuestas: la primera corresponde a la utilización de una teoría base de análisis cualitativo como la Teoría Fundamentada, de Bamey Glaser y Anselm Stra uss, ${ }^{24}$ identificando de esta manera las prácticas sociales de los sujetos; luego para comprender las prácticas disc ursivas y textual se hizo una 
25 J ÄGER, 2003.

${ }^{26}$ Luis Enríque ALONSO, 2003, p. 204.

27 CONNELL, 1998; OLAVARRÍA 2001a, 2001b, 2005; María José MORENO Ruiz, 2001.

28 OLAVARRÍA, 2005. aproximación de acuerdo a la propuesta de ACD de Siegfried Jäger. ${ }^{25}$ Esta a proximación implicó rescatar los elementos más significativos y pertinentes para el estudio. Por lo tanto, nos centramos en id entific ar el hilo disc ursivo, su caracterización y la combina ción de un a nálisis global y fino de la textualidad de los discursos de los hombres.

Estas p ropuestas de a ná lisis fueron a p rop ia da s pa ra la investigación, ya que genera ron resulta dosque describen el fenómeno y permiten establecer relaciones teóricas. Se trató de situar los discursos no sólo en consideración al marco situacional, sino que también a los propios sujetos y sus subjetivida des. ${ }^{26}$

\section{Resultados y análisis: hilando los discursos}

Casi dos décadas después de los avances experimentados en las sociedades ind ustria liza das, se inician los estudios sobre la masculinidad en Chile, sobre todo los referidos a la construcción de la identidad de género de los hombres. Sirviéndose de los adelantos ya alcanzados en otros contextos, ha comenzado un interés creciente en distintas universidades y centros de estudios del país porabordar interrogantes sobre sí la masculinida d se encuentra en crisis, cómo se construye hoy la masculinidad. ¿Hay una nueva masculinidad?

Más que responder estos cuestionamientos exha ustiva mente abordados por diferentes autore $s^{27}$ concluyen sobre las transformaciones que se han experimentado en las identidades de género y que están estrechamente vinculadas a procesos estructura les como la globalización, la sociedad de la información y el modelo neoliberal. Asimismo, los estudios realizados en $C$ hile expresan que, aunque los hombres se muestran proclives a las demandas de las mujeres, en la vida cotidiana no se a dvierten cambios signific a tivos, ya que ellas siguen siendo lasprincipales responsables de lo doméstic o y de la crianza de los hijos. Igualmente se confirma una cierta fragilidad en los discursos que hablan de una nueva masculinidad, ya que no tienen un correlato empírico que los sustente. Más aún, a spectos como la intensidad del lazo amoroso tendría un papel preponderante en la relación de pareja, de manera que facilitaría negociar de mejor manera la participación del varón en la unidad doméstica. ${ }^{28}$

A partir de estos hallazgos, nos centramos, en aquellas transformaciones de la masculinidad que han provocado una ruptura en la exclusividad de los espacios de poderque históric a mente eran a signadosa los hombres, tanto en el ámbito público como en el privado, configuránd ose nuevas formas de relaciona rse en el interior 
${ }^{29}$ Kathia ARAUJ O y Francisca ROGERS, 2000, p. 60.
30 Irene VASILASC HIS DE GIALDINO, 2003. de la familia, en el ámbito laboral y en las distintas esferas de sociabilidad. Estos procesos de fracturas sociocultura les han modific ado los equilibrios previos, surgiendo nuevos órdenes de género en los que se cuestionan algunos valores como la masculinidad hegemónica, el autoritarismo, la violencia intrafamiliar, entre otros.

Estas variaciones han sido referidas por algunos autores como una ruptura de ciertos sustentos estructura les que se acompañan por la pérdida de los significados de la masculinidad, develand o distintas formas de ser hombre, ampliando las identidades y las formas de construir lo masculino..$^{29}$

Desde aquíse propuso en este estudio hacerun giro respecto a la tendencia que se ha dado en las investigaciones de género, en el sentido de abordar estas temáticas generalmente en forma dicotómica, ya sea lo femenino o lo masculino. En este sentido buscamos destacarel ca rácter relacional de los cambios, ya que han desencadenado modificaciones en la construcción de identidades de hombres y mujeres marcadas por las transgresiones, rupturas resistencias de género, que están lejos de lograr un acomodo social, por lo tanto, avanzar en su análisis posibilita su abordaje y comprensión.

Dicho esto, a continuación presentaremos una síntesis sobre algunos resultados del estudio que se a proximan a delinearlastensiones en los espacios de poder y liderazgo femenino, desde la textualidad masculina.

\section{Los caminos zigzagueantes de las mujeres al poder: entre el control y la tensión}

En los disc ursos a na liza dos, tienen como elemento transversal la dificultad de los hombres para hablar desde el yo; la tendencia es que ellos se explayan en un lenguaje general que no los interpela en sus experiencias individuales. El hilo discursivo que cruza el habla masculina está marcado por una serie de oposiciones ${ }^{30}$ personal/impersonal, partic ula r/g enera l a fima tivo/neg a tivo, gusto/disg usto, aceptación/rechazo. Esto queda de manifiesto en formula ciones ambivalentes respecto a las percepciones que tienen sobre el poder alcanzado por las mujeres en los ámbitos laborales, polític os y fa milia res.

Los hombres elaboran un discurso proactivo, favorable al cambio de roles que se ha experimentado en la sociedad chilena, que ha impulsado la incorporación de las mujeres al mercado laboral, pensando en las transformaciones como un proceso normal de evolución hacia un mayor protagonismo de las mismas. 
${ }^{31}$ Entrevista individual, hombre 62 años.

32 María Asunción GONZÁLEZ DE CHÁVEZ Femández, 1998, p. 16.

${ }^{33}$ Entrevista individual, hombre 60 años.

${ }^{34}$ Entrevista individual, hombre 55 años.

5 Paulina SALINAS y Susana ARANCIBIA, 2006.
Las mujeres estaban relegadas a la casa y no podían votar, no tenían ninguna participación ciudadana, no tenia ningún tipo de decisión cívica, era difíc il que ellas llegaran a accederal poder, pasan los años y la mujer se comienza a incorporara la fuerza laboral, más aún de manera más formal, accede más a la educación, antes era muy difíc il. ${ }^{31}$

Los conflic tos de las mujeres de hoy están vinc ula dos con las restric ciones impuestas al modelo de mujer/ feminidad vigente, con las dific ulta des que presentan ellas para descubrir/aceptar/expresar sus deseos al margen de dicho modelo, y con la vigencia de una organización social y una identidad masculina, compartida por las mujeres, que no permite el cuestiona miento real del sta tu quo, al no integrar como responsabilidad y (dis) valores "femenino", estigmatizados y/o hiperdemandado a las mujeres que luchan por un nuevo modelo social y de género. ${ }^{32}$

Como ejercen el poder hoy día las mujeres... yo creo que siempre, sin perder su rol femenino y yo creo que eso les ayuda mucho, yo te diría esa coquetería innata que tiene la mujer, no puede perderla; a unque pudiera mandar, porque ya me veo una niña buenamoza te fijas, y es la jefa más encima. ${ }^{33}$

Ahora hay mujeres que han alcanzando, cargos que antiguamente ni siquiera se habían soñado. Pienso que las mujeres son muy capaces en los puestos son más metódicas, son, "menos comuptas" que los hombres, desde mi punto de vista personal, yo creo que están entrando en una esfera donde ya no deberían haber muc hos disting os de sexo. ${ }^{34}$

Junto a esta visión proactiva al cambio de roles de las mujeres en la sociedad se devela en los discursos también la vigencia de mandatos socioculturales que las constriñen en un imaginario social masculino de: mujer/ madre/feminidad, configurándose, entonces, un proceso dinámico, transformador, con elementos de control y tensión, ruptura y continuidad en las relaciones entre los géneros. En otras palabras, en los discursos se constata la vigencia de la perspectiva esencialista en los hombres, y que circunscribe a estas últimas a una identidad de mujer, pasiva sensible, femenina, correspondiente con una identidad homogénea construida desde la estructura patriarcal y que retrotrae a las mujeres al espacio privado como principales responsables de las ta reas doméstic as y reproductivas, y con una marcada distribución sexual de los roles. ${ }^{35}$

Mi esposa hace poco se puso a trabajar, hace poco, yo creo que hace un año más o menos. La percepción 
${ }^{36}$ Entrevista grupal № 2.

${ }^{37}$ Entrevista g rupal № 2.

${ }^{38}$ Entrevista grupal № 3.

${ }^{39}$ SALINAS y ARANC IBIA, 2006.

${ }^{40}$ G ONZÁLEZDE CHÁVEZ, 1998, p. 15. que tengo de este asunto, personalmente, hemos tenido a lgunos problemas por el asunto, por el problema de que ahora que ella... aparte del dinero que yo le entrego, ella ahora recibe su dinero cierto, pero ahora por ejemplo a veces tenemos conversaciones, y ella como que se pone muy altanera, o sea como que a hora como que dispara más de lo que se disparaba antes. Yo creo que como se puede decir vulga mente, como que se creen el cuento. ${ }^{36}$

Tiene su pro y su contra que la mujer trabaje. Yo ahora estoy teniendo problemas por ese asunto, porque yo siempre llevé el dinero a la casa, cierto yo, o sea, no es que ella me pase a llevar, sino como que ella tiene otra percepción de este asunto; diga mosahora, según, como que se pone más altanera, así ya, me levanta más la voz, y uno que es..., yo me considero un hombre machista, para mi es molesto. ${ }^{37}$

Por mi parte, no me gustaría que la mujer trabajara o sea, yo no soy machista, pero yo creo que el hombre es el que, que las lleva en la casa, uno le da alimento a ella, porque la mujer cría los hijos. ${ }^{38}$

Esta resistencias, como decíamos, se tra sla pan con disc ursos má s p rog resista s en los va rones que se a venturan hacia un reconocimiento de lascapacidadesy nuevos roles que han ido asumiendo las mujeres en el espacio público, aunque se trata de un reconocimiento vigilante, a mbivalente, ya que está mediatiza do poruna percepción de confianza/desc onfianza, eficiencia/incompetencia, hacia lo que ellas son capaces de lograr, lo que, muchas veces, también es autoreferido por las propias mujeres. ${ }^{39}$ De ello se infiere la actualidad que tiene en la identidad femenina una construcción en la que preexiste una natura leza o una esencia femenina o masculina, subsidia ria de un orden simbólico dado, también construido, y, por tanto, no único posible e inmodificable. Tales identidades van enraizadas a un sistema de representaciones y a elaboraciones culturales que sirven de base y las cimentan. ${ }^{40}$

Esta s rep resenta ciones a mbig uas tienen a ltos costos afectivos y emocionales para las mujeres y que redundan en insatisfactorias relaciones inter género y que por su naturaleza son de difícil contención. En el ámbito privado ellas se articulan, ya sea cediendo, traspasando o negando sus propias a spira ciones. Y en el ámbito público, espacio de desarrollo en el que se juegan aspiraciones como el reconocimiento de los otros, el respeto, la valoración, son logros que alcanzan a costa habitualmente de otros aspectos de su vida, la familia, la relación con los hijos, con la pareja, ya que estos últimos y las propias mujeres 
${ }^{41}$ GONZÁLEZ DE CHÁVEZ, 1998.

${ }^{42}$ Entrevista grupal № 2. responden al imaginario social de la mujer como sujeto capaz de cubrir todas demandas y con alta capacidad, para estar simultá nea mente cubriendo "exitosamente" las distintas demandas del espacio público y privado.

A pesar de la mayor apertura del campo laboral para las mujeres, ellas se encuentran en dificulta des para compatibilizar el papel tradicional que de ellas se espera en el hogar con la inclusión en un medio cultural androcéntric 0 . Ya que este ámbito a lta mente competitivo ha sido organizado por el hombre para cumplir con el nivel de exigencia acorde con las circunstancias, contando con el contingente provisto por la mujer para cubrir las necesidades vitales y emocionales de él y de los hijos.

En consecuencia, la tensión que experimentan las mujeres en la configura ción de su identidad y en el proceso de empoderamiento como mujeres y como madres, es donde se plasma con mayor énfasis el conflicto y las resistencias, llenando de sentimientos de culpabilidad e inadecuación a aquéllas que se desmarcan de él o comparten dura mente diversa sta rea sintra y extra fa milia res. Por otra parte, muchas madres, gratificadas a menudo en detentar el lugar - la fantasía - de la omnipotencia, han tenido y tienen dificultades internas y sociales para escaparse al papel y la imagen en que han sido encerra das. ${ }^{41}$

Por ejemplo, el cambio también lo que yo me he dado cuenta también que por ejemplo mis niños cierto y a veces yo me he levantado y los niños tienen puesta la mesa digamos, las tacitas todo, la tetera y todo el asunto, o sea como que ellos también se están acostumbrando a ese sistema pero también les ha afectado, por ejemplo la niña no hace tareas hasta cuando llegue la mamá, el más chiquito también, tiene que hacer algún trabajo y todo, lo hace, pero está esperando a su mamá. ${ }^{42}$

Yo creo que hay dos cosas, que trabaje la mujer es bueno, porque también tiene derecho igual que el hombre a estudiar, a desarrollarse profesionalmente todo el asunto, pero a la vez también es un atentado contra la familia porque alguien tiene que estar más, como cerca con los hijos. Son dos cosas, una que la mujer se pueda desarrollar profesionalmente y otra ¿pero qué pasa con la familia? ¿qué pasa con loshijos?, $y$ todos estos problemas que se han suscita do a hora. ${ }^{43}$

El modelo de mujer-madre conserva toda su vigencia étic a, en los disc ursos masculinos, llena ndo de sentimientos contra dic torios las existencias femeninas. Hay un deseo de la matemidad a rraigado a la socialización primaria de las mujeres, y que encuentra un cimiento idealizador en los 
${ }^{44}$ Entrevista grupal № 4.

${ }^{45}$ Entrevista grupal № 4.

${ }^{46}$ Entrevista individual, téc nic 0,20 años. hombres. Porlo tanto para aquéllas que desean distanciarse de esta construcción resulta culpabilizador, complejo cargado de dificulta des internasy sociales, ya que implica negar un imaginario social que ha sostenido la familia nuclear patriarcal.

Aparte que de todo esto del cambio, también la mujer es... una... parte súper importante, es protagonista, porque en estos tiempos igual, hay mujeres que dic en, por ejemplo tú dic es ya, voy a lavar, voy a cocinar, voy a hacer esto otro y la mujer te dice no, yo hago eso, no, yo plancho, entonces como que la mujer también se siente a menazada porque tú estay ocupando el rol que ocupa ella. Entonces ahí la mujer cumple una función principal y si realmente queremos lograr un equilibrio y una igualdad la mujer también va a tener que cambiar la mentalidad.$^{44}$

Hay bastantes mujeres que aunque trabajen y todo, tienen una mentalidad machista, o sea al hombre no lo dejan hacer sus cosas y lo terminan haciendo ellas dándole en el gusto en todo. ${ }^{45}$

En los discursos los varones hablan de los nuevos roles que las mujeres desempeñan en la sociedad actual, sobre todo en el ámbito laboral y la compatibilidad de ellos, pues a prueban que la smujeres trabajen y a porten al sustento de la familia, pero ojalá esto no modifique el ord en familiar y por ende, a unque ellos ya no sean los único proveedores, sus expectativas se aferran a los mandatos de género de la familia tradicional, respecto a la crianza y cuidado de los hijos.

Es que antes eran distintos los cambios, la sociedad era distinta, o sea, con la mujer tenía muchos más hijos que ahora, y era por la circunstancia, ante se podía tenermás hijos, ahora ya no se puede tenertantos hijos como antes, entonces la mujer debe tomar otros roles también más protagónicos en la parte laboral, para poder mantener un equilibrio económic 0 , porque ya no alcanza a lo mejor con un sueldo, o simplemente que la mujer sea dueña de casa, ya no basta con por la política económica que actualmente estamos viviend $0 .{ }^{46}$

La participación social igual ha ido en menoscabo un poco de la participación que las mujeres tenían en la crianza de los hijos antiguamente. Hoy en día, la mujer pasa mucho tiempo fuera del hogar como que ha memado, en cierta forma las relacionesfamiliares, pero igual es positivo que esté trabajando, esté ocupando cargos, porque igual posee capacidades...que son bien positivas también, en todas las cosas existen ventajasy desventajas. Y la desventaja es esa, la crianza 
${ }^{47}$ Entrevista grupal № 4.

${ }^{48}$ María Elena VALENZUELA, 2000.

49 Rosalía TODARO, Amália MAURO y Sonia YAÑEZ, 2000, p. 94. de los hijos ya se le está delegando a la escuela, a los profesores, a las academias, no sé, a los gimnasios, a losclubesdeportivos... ya no es tanto la casa, la familia, como el núcleo de la formación de una persona. ${ }^{47}$

En las últimas décadas han surgido nuevos modelos productivos que han a fec ta do signific a tiva mente el sistema de relaciones laborales y la calidad de los empleos. Estos modelos tienen como centro la flexibilidad del empleo y de la organización de las empresas, y han brotado en medio de una masiva incorporación de las mujeres a la fuerza de trabajo. En el Cono Sur, Chile registró el mayor incremento en los últimos 30 años, a un cuando el nivel de participación laboral de las mujeres se sitúa bajo el promedio regional. ${ }^{48}$ Sin embargo, la incorporación masiva de las mujeres al empleo ha sido acompañada de una notable precarización del mismo. Aspectos de la calidad del empleo de las mujeres, tales como la regularidad y estabilidad laboral, los ingresos, las horas de trabajo y su intensidad, los peligros ocupacionales para la salud, el ambiente de trabajo, la protección laboral y social, los contenid os de los empleos y las oportunida desque ofrecen para el desa rrollo personal y las tra yec toria slabora les, como el esta tus social que proporciona, están siend o fuertemente permeados por el fenómeno de la segregación de género. ${ }^{49}$

Aunque la identidad masculina tradicional sigue vigente, se aprecian transformaciones en su forma y en su fondo, ya no se trata del hombre que exige abiertamente que la mujer permanezca en el espacio doméstico y que realice las ta reas que históric a mente se le han asigna do al sexo femenino, vale decir, socialmente se reconoce que la smujeres deben c umplir un rol más a ctivo en la sociedad, tener un trabajo y realizarse como persona. Sin embargo, el histórico machismo se ha camufla do y hoy se basa más en el control y en la coerción psicológica que en la discriminación o en las restricciones físicas. Es el dominio que se ejerce en la clandestinidad, el denominado neomachismo.

Sabe una cosa yo pienso que cuando, que las mujeres se ahogan mucho de que cuando se habla mucho de que se ha incorporado al ámbito laboral. Es cierto si hay mujeres de que se han superado y han... han digamos ehh... al hombre lo han superado digamos, en muchas, en muchascarreras, muchas etapas, pero si es cierto entra a una empresa y empiezan a discriminarla, me entiende, a a islarla, o sea siempre el hombre va pornaturaleza, va a tirarla pal lao... Porque es así, yo lo veo de esa manera, no porque soy machista ni nada de eso, por naturaleza se da así, se da así, la 
${ }^{50}$ Entrevista grupal № 2.

${ }^{51}$ Entrevista grupal № 2. mujer... llega una jefa y el jefe de al lado y... le hace la guerra y ella no tiene nada que vercon los empleados es una guerra de jefatura digamos. ${ }^{50}$

Me ha afectado bastante esta situación de que la mujer trabaje, porque como tú siempre dices que tú vas a aportar siempre y ahora que te sientes con un poco menos derechos de repente, que tienes que llegar como de otra manera. ${ }^{51}$

Si bien la participación de loshombresen el espacio doméstic o ha aumentado, presenta a lgunas restric ciones: primero los hombres conciben su participación como un apoyo generoso a la mujer, es decir, está condicionada por el lazo afectivo que estos tengan con la pareja y el éxito que logren las mujeres en las negociaciones cotidianas, vale decirno es el resultado de una convic ción cabalde éstos por ha cerse cargo de las ta reas doméstic as por el solo hecho que son parte de un grupo familiar. Segundo, este apoyo también se limita a algunas tareas dentro de la estructura familiar, de hecho los hombres se toman la opción de elegirqué hacen y qué no, en cambio para las mujeres esta opción no existe.

\section{Los hilos disc ursivos}

Al analizar lo discursos en forma longitudinal y transversal se pueden distinguir tópicos centrales y que se presentan en oposición respecto al poder alcanzado por las mujeres, y los obstáculos que visualizan ellos en el proceso de empoderamiento.

- Observan una transformación social, un cambio de mentalidad respecto a los roles que desempeñan actualmente las mujeres en la sociedad.

- La globalización implica el poder tener acceso a otras culturas distintas donde hay más tolerancia, y así a lejarse del pensamiento religioso y tener más a pertura.

- Con la llegada de la democracia se han abierto más espacios para la mujer, que está adquiriendo cada vez más poder.

- Los hombres a prueban el discurso oficial respecto a la igualdad de derechos que tienen hombres y mujeres en la sociedad.

- El hombre ejerce el poderporel poder, las mujeres ejercen el podery le agregan las relaciones humanas y el trato.

- Las mujeres son las que manejan las relaciones personales en todos los ámbitos.

- Las mujeres son más capaces, más metódicas, menos comuptas, más delic adas y meticulosas. 
- Son relevantes los ingresos económicos que generan actualmente las mujeres para el bienestar de la familia.

- Valoran positivamente que las mujeres sean un par con la cual se puede conversary que no deben responder a las expectativas de lo que se considera "un macho proveedor".

- En la familia la mujer ejerce el poder.

- Hay resistencia de parte de las mujerespara ceder el poder al interior de la familia.

- Las actividades propias del hogar deberían ser compartidas, no puede seguirse cargando la mano a una sola persona (mujer).

- Las mujeres se están incorporando en diferentes áreas y no se sabe si el mercado logrará absorber toda esa mano de obra.

- La mujer está preparada para hacer lo que quiera, pero incomoda.

- En lospuestos de ministros hay paridad, sin embargo no se sabe si serán capaces como los hombres en puestos $\tan$ a ltos.

- Para los hombres que a spiran al poder, consideran a la mujercomo una persona que se le cruza en el camino.

- Las mujeres no deben perder sus características innatas, ni su rol femenino.

- Las mujeres tienen que demostrar más que los hombres para poder ejercer liderazgo, tienen que tener más cuidado, los hombres pueden hacer más cosas, que a las mujeres no se les perdonaría.

- Los hombres están siendo igual que las mujeres y por eso surgen problemas en las relaciones de pareja.

- En el norte del país el poder masculino ha sido emblemático, porserdesc endientes de la cultura pampina, donde la virilidad masculina se medía con el poder absoluto.

- La asunción de Michelle Bachelet a la presidencia de la república significo una perdida importante para los hombres.

- Las mujeres se desenvuelven bien en los centros de madres, en las juntas de vecinos, son cargos que debieran llevar ellas por natura leza, porque es un centro social que tiene la facultad de ocuparse de la comunidad $y$ de los niños.

- A las mujeres les cuesta más llegar al poder, no tienen plataforma, eso hace mucho en el tema del liderazgo. 


\section{A modo de conclusión}

${ }^{52}$ FAIRCLOUG Y Y WODAK, 2000.

En base a tres de los principios teóricosmetodológicos del $A C D^{52}$ a nalizaremos, a modo de conclusión, cómo se relaciona cada uno de ellos con el a ná lisis crítico rea liza do sobre los discursos masculinos.

- Las relaciones de poder como elementos discursivos, el ACD subraya el carácter discursivo de las relaciones sociales de poder en la sociedad contemporánea, carácter que proviene en parte de cómo se ejerce y negocian las relaciones de poderen el interior del discurso. Al respecto, el habla de los hombres devela el malestare incomodidad que le produce la incorporación de la mujeres a los espacios de poder que históric amente han sido ocupados por ellos, sean estos en el mercado laboral o en los espacios de decisión, ministerios, presidencia de la república, entre otros. Los hombres refuerzan en sus disc ursos la vig encia de las característic as esencialistas de las mujeres, la femineidad, la responsabilidad que se le asigna en la manutención de las relaciones de pareja y su preocupación porlas actividades de la comunidad y de los niños.

- El discurso modela la sociedad y la cultura, y esto implica que el lenguaje hace su propia contribución a la reproducción y/o transforma ción de la sociedad y la cultura, incluidas las relaciones de poder. En los hilos discursivos id entific a dos en las entrevistas se observan con intensidad elementos de continuidad y de cambio en los discursos masculinos, es decir, el proceso argumentativo en la producción del texto evidencia una serie de oposiciones c a pacidad/incapacidad, más participación/menos liderazgo, menos comuptas/sin pla ta forma política, etc. Ha y a mbigüedad y un cierto continuum en las representa ciones que hacen los hombres sobre las mujeres, donde se da una dialéctica entre características tradicionales y modernas, esta súltima s reconocidas como eminentemente masculinas.

- El discurso es histórico, y esto implica que se produce en un contexto, ha y convenciones y reglas subyacentes que es necesario reconocer como asimismo los elementos del pasado que nos remite el discurso. En este sentido podemos señalar que la ambigüedad y tensión disc ursiva a na liza da es concomita nte con el proceso identita rio de la sociedad chilena actual, que se caracteriza por la existencia de profundas transforma ciones que conjugan aspectos tradicionales con otros más modemizadores, es decir, en el proceso identitario de la sociedad chilena hay una coexistencia de diversas dimensiones que van confor-

${ }^{53}$ J orge LARRAíN, 2001. mando un proceso dinámico y en construc ción..$^{53}$ Lo a nterior 
se manifiesta, por ejemplo, en que se mantiene una tradic ión de a utorita rismo en la s rela ciones junto con una valoración de relaciones más democráticas; hay una mayor a pertura produc to de la globaliza ción y el mayor conta c to con el resto del mundo, pero también existe un racismo más bien oculto, sobre todo con los países vecinos. El machismo tiene plena vigencia en algunos segmentos de la sociedad, mientras que en otros se encuentra más camufla do. Cada una de estas dimensiones nos permite visua lizar la ambigüedad y sincretismo presentes en el proceso modemizador que experimenta la sociedad chilena.

Finalmente, las trayectorias de las mujeres hacia la autonomía y el posicionamiento en el ámbito público son zigzagueantes en el sentido que están plasmadas por tensiones entre las resistencia s que provoca n en el espacio intra y extra familia, como por las oportunidades que han sido capaces de ir generando en la sociedad. Esta oposición marca el desempeño y posicionamiento de las mujeres en los distintos planos del quehacer nacional.

En los discursos a naliza dos queda en evidencia las tensiones que están vivenciando los hombres respecto al empoderamiento femenino. Hay reconocimiento y temor, hay seguridad frente al desempeño y capacidades de las mujeres, pero al mismo tiempo incomodidad y el deseo a que las mujeres manteng an sus ca rac terístic as "na tura les", de femineidad, sensibilidad, etc.

Los obstá culos que refieren los hombres respecto al empoderamiento de las mujeres se relacionan con que las limitantes que se les pueda generar a ellos en aquellos espaciosdonde han tenid o control, el trabajo o la actividad política.

Analizar los discursos y el habla, en este caso masculina ha posibilitado, desmontar las estructuras esencialistas presentes en el lenguaje que producen y reproducen prácticassocia les discrimina torias, y que se van reforzando en el imaginario social de los sujetos.

\section{Referencias bibliográficas}

ALONSO, Luis Enríque. La mirada cualitativa en sociología. Una aproximación interpretativa. Madrid: Editorial Fundamentos Colección Ciencia, 2003.

ARAUJ O, Ka thia; ROGERS, Francisca. "El Hombre: ¿Existe?" En: OLAVARIA, J osé; PARRINI, Rodrigo (Eds.). Masculinidad/es. Identidad, sexualidad y familia. Primer Encuentro de Estudios de Masculinidad. Santiago de Chile: FLAC SO-Chile/Universidad Aca demia de Humanismo Cristia no/Red de Masculinidades, 2000. p. 59-66. 
CONNELL, Robert W. "El imperialismo y el cuerpo de los hombres". En: VALDÉS, Teresa; OLAVARRÍA, J osé (Eds.). Masculinidades y equidad de género en América Latina. Santiag o de C hile: FLACSO, 1998. p. 76-89.

FAIRC LOUGH, Norma; WODAK, Ruth. "Análisis critic o del discurso". En: VAN DIJ K, Teun A. (Comp.). El disc urso como interacción social. Estudios sobre el discurso II. Una introducción multidisciplinaria. Barcelona: Editoria I Gedisa, 2000. p. 367-404.

GIM - Grupo Iniciativa Mujeres. El Nuevo Contrato Social Dirigido a las Mujeres. Balance de una década de democracia en Chile. Santiago de Chile: Gim, Cedem, Cem, La Morada, Corsap, Domos, Flacso, Fundación Instituto de la Mujer, Isis Internacional, Mench, Prosam, 2002.

GONZÁLEZ DE CHÁVEZ Fernández, Ma ría Asunción. Feminidad y masculinidad. Subjetividad y orden simbólico. Madrid: Biblioteca Nueva, 1998.

HENNECKE, Angelika. El discurso como flujo de conoc imientos a tra vés del tiempo. Córd oba, Argentina: Comunic arte Editorial., 2004.

HTUN, Mala. Mujeres y poder político en Latinoamérica. International Institute for Democracy and Electoral Asístanse, 2003. Documento de la Web: www.idea.int/ public ations/wip/upload/chapter_01-2.pdf .

INE - Instituto Na cional de Estadísticas de Chile. Censo 2002. Documento de la Web: www.ine.cl/cd2002/ sintesisc ensa l.pdf.

IÑIGUEZ Rueda, Lupicinio. "El análisis del discurso en las ciencias sociales: variedades, tra diciones y práctica". En: IÑIG UEZ Rueda, Lupicinio (ed.). Análisis del disc urso. Manual para las ciencias sociales. Barc elona: Editorial UOC, 2003. p. 83-123.

J ÄGER, Sieg fried. "Disc urso y conocimientos a spectos teóric os y metodológic os de la crítica del disc urso y del a ná lisis de disp o sitivos". En: WODAK, Ruth; MEYER, Mic a el (Coords.). Métodos de análisis crítico del discurso. Barcelona: Editorial Gedisa, 2003. p. 61-99.

LARRAÍn, J orge. Identidad chilena. Santiag o de C hile: Lom Ediciones, 2001.

MACHICAO Barbery, Ximena. "Algunas reflexiones sobre el contexto, paridad y desafíos para los movimientos feministas". Revista Con-spirando. Revista Latinoamericana de Ecofeminismo, Espiritualidad y Teología, Santiago de Chile,. n. 52, p. 20-27, abril 2006.

MORENO Ruiz, María José: "Masculinidades en la cultura de la globalización". En: OLAVARRIA A., José (Ed.). Hombres: identidades y violencia. 20 Encuentro de Estudios de Masculinidades: Identidades, Cuerpos, 
Violencia y Polític as Públic as. Sa ntia go de Chile: FLAC SOChile/Universidad Academia de Humanismo Cristiano/ Red de Masculinidades, 2001. p. 37-54.

OIT- Organización Intema cional del Trabajo. ¿Más y mejores empleos para las mujeres?. La experiencia de los países del MERCOSUR y Chile. Editores: María Elena Valenzuela y Gerhard Reinecke. Santiago de Chile: OIT, 2000.

OLAVARRÍA, José. "De la identidad a la política: masculinidades y política públicas: auge y ocaso de la familia nuclear patriarcales en el siglo XX". En: OLAVARRÍA, J osé (Ed.). Hombres: identidad/es y violencia. Encuentro de Estudios de Masculinidades: Identida des, Cuerpos, Violencia y Polític as Públicas. Sa ntia go de Chile: FLAC SO-Chile/Universidad Academia de Humanismo Cristiano/Red de Masculinida des, 2000. p. 11-28.

OLAVARRÍA, J osé. "Los va rones heterosexuales: sexualidad, deseo y placer". En: OLAVARRÍA, José. ¿Hombres a la deriva? Poder, trabajo y sexo. Santia go de Chile: FLACSO, 2001a. p 39-88.

O LAVARRIA A., J osé (Ed.). "Ho mb res: id entid a desy violenc ia". En: 2을 Encuentro de Estudios de Masculinidades: Identidades, Cuerpos, Violencia y Políticas Públicas. Santia go de Chile: FLAC SO, 2001b.

OLAVARRÍA, José. “¿Dónde está el nuevo padre? Trabajo doméstico: de la retórica a la práctica". En: Seminario "Familia y Vida Privada ¿Transformaciones, Tensiones, Resistencias y Nuevos Sentidos?" Santiago de Chile: FLAC SO-C hile, CEDEM, UNFPRA, 2005. p. 215-250.

PNUD - Programa para las Naciones Unidas para el Desa rrollo. Desa rrollo Humano en Chile. El Poder: ¿Para qué y para quién? Santiago de Chile: PNUD, 2004.

RUIZ, Olga; SOLANO, Sandra; ZAPATA, Claudia. "Redes de mujeres pobladoras de la comuna de San Joaquín. Participación y ciudadanía emergente". Revista Proposiciones, Sociedad Civil, Participación y Ciudadanía Emergente, Santiago de Chile: SUR Ediciones, n. 28, p. 213-230, 1998.

RUIZ, José Ignacio. Metodología de la investigación cualitativa. Biba o, España: Universida d de Deusto, 1999.

SADín Esteban, María Paz. Investigación cualitativa en educación. Fundamentos y tradiciones. Madrid: Mc Graw Hill, 2003.

SALINAS, Paulina. "Ciuda da nía y género. Mujeres y liderazgo en las Provincias de Antofagasta, El Loa y Tocopilla". Revista Electrónica de Trabajo Social. Public ación a nual editada por el Departamento de Servicio Social, Facultad de Ciencias Sociales, Universidad de Concepción, Chile, p.1-4, 2002. 
SALINAS, Pa ulina. "Asoc ia tividad y género: construcc ción del liderazgo femenino en la región de Tarapacá y Antofagasta". Revista Salares, Universidad de Antofagasta, año 7, n. 5, p. 79-98, 2005.

SALINAS, Pa ulina; ARANCIBIA, Susana. "Disc ursos ma sc ulinos sobre el poder de las mujeres en Chile. Sujetos y subjetividades". Revista Ultima Década, Valparaíso, Chile: Centro de Estudios Soc ia les CIDPA, a ño 14, n. 25, p. 65-90. 2006.

SCOLLON, Ron. "Acción y texto: para una comprensión conjunta del lugar del texto en la (inter) acción social, el análisis mediato del discurso y el problema de la acción social". En: WODAK, Ruth; MEYER, Micael (Coords.). Métodos de análisis critico del discurso. Barcelona: Editorial Gedisa, 2003. p. 205-266.

SERNAM - Servicio Nacional de la Mujer. Plan Regional de Igualdad de Oportunidades entre Mujeres y Hombres 2000-2010, Región de Antofagasta. Antofa gasta, Chile: SERNAM, 2000.

SERNAM - Servic io Nacional de la Mujer. Detec ción y a nálisis de la prevalencia de la violencia intrafamiliar. Santiago de Chile: SERNAM/Universidad de Chile. Centro de Análisis de Polític as Públicas, 2001.

STRAUSS, Anselm; CORBIN, Juliet. Bases de la investigación cualita tiva. Téc nic as y procedimientos para desarrollar la teoría fundamentada. Medellín, Colombia: Contus/ Editorial Universidad de Antioquia, Facultad de Enfermería de la Universidad de Antioquia, 2002.

TODARO, Rosalía; MAURO, Amalia; YAÑEZ, Sonia. "Chile: la calidad del empleo. Un a nálisis de género". En: VALENZUELA, Ma ría Elena; REINEC KE, Gerhard (Eds.). ¿Más y mejores empleos para las mujeres?. La experiencia experiencia de los países del MERCOSUR y Chile. Santiago de Chile: OIT, 2000. p. 193-265.

VALDÉS, Teresa; WEINSTEIN, Mariza. Mujeres que sueñan: las organizaciones de pobladoras 1973-1989. Santiago de Chile: FLACSO, 1993.

VALDÉS, Teresa (Coord.). El Indice de Compromiso Cumplido-ICC. Una estrategia para el control ciudadano de equidad de género. Santiago de Chile: FLAC SO-Chile, 2001.

VALENZUELA, María Elena. "La situa ción la boral y el acceso al empleo de las mujeres en el Cono Sur". En: OIT Organización Internacional del Trabajo. ¿Más y mejores empleos para las mujeres?. La experiencia de los países del MERCOSUR y Chile. Santiago de Chile: OIT, 2000. p. 9-27. 
VASILAC HIS DE GIALDINO, Irene. Pobres, pobreza, identidad y representaciones sociales. Ba rc elona: Ed itorial Gedisa, 2003.

VALLES, Miguel S. Técnicas cualitativas de investigación social. Reflexiones metodológicas y práctica profesional. Madrid: Editorial Síntesis, 2000.

VAN DIJ K, Teun A. Racismo y análisis crítico de los medios. Paidós: Ba rcelona, 1997.

WODAK, Ruth. "De que se tra ta el a ná lisis crític o del discurso (ACD). Resumen de su historia, sus conceptos funda mentales y sus desa rrollos". En: WODAK, Ruth; MEYER, Mic a el (Coords.). Métodos de análisis crítico del discurso. Barcelona: Editorial Gedisa, 2003. p. 17-59.

[Recebido em novembro de 2006 e aceito para publicação em fevereiro de 2007]

\section{Masculine Discourses as Control and Tension Devices in Women's Lea dership and Empowering Configuration}

Abstract The present contribution deals with the meaning of the feminine leadership from a masculine perspective. By using a qualitative approach, open interviews were developed to und erstand the meaning that men assign to the feminine leadership. The speec hes were a nalyzed on the basis of the Strauss/C orbin-theory (2003). The results show that men a gree with the changes experienced by the gender roles. In a specific way, men agree that women need to have more soc ietal-ec onomic op portunities, which are nec essa ry for more equitable rela tionship inside the family. However, within this positive framework emerging tensions configure a dissatisfaction of the masculinity that, in turn, impact the intima te sphere questioning the present day inter-gender relationship. Finally, there is a men's group with more progressive speeches which venture towards a positive evaluation of the women's capacities and their assumed public space-roles. However this positive evaluation is vigilant, a mbivalent, and influenced by a perception of confidence/ unc erta inty, effic iency/ineffic iency of the effective soc ietal contribution that women a re able to reach.

Key Words: Power; Societal Changes; Gender. 